\title{
Macroscopic response of regular masonry from homogenization: comparison of isotropic and orthotropic damage models
}

\author{
Tomáš Krejčć1 ${ }^{1}$, Tomáš Koudelka ${ }^{1}$, Vasco Bernardo ${ }^{2}$, Michal Šejnoha, ${ }^{1, *}$ \\ ${ }^{1}$ Department of Mechanics, Faculty of Civil Engineering, Czech Technical University in Prague, Thákurova \\ 7, 16629 Prague 6, Czech Republic \\ ${ }^{2}$ National Laboratory of Civil Engineering, Avenida do Brasil, 101 1700-066 Lisboa, Portugal
}

\begin{abstract}
This paper outlines prediction of macroscopic effective properties of a regular masonry from homogenization. It focuses on the derivation of nonlinear macroscopic stress strain curves adopting either classical isotropic or more advanced orthotropic damage model. The response resulting from both tensile and compressive uniaxial loading is examined in the light of strain and stress loading regimes. A masonry structure typical of "Placa" buildings (mixed masonry- reinforced concrete buildings) built in Portugal is selected as one particular example to illustrate the differences in the predictive capabilities of the two constitute models on the one hand and the formulation of the homogenization problem on the other hand. It is suggested that the mixed loading conditions are essentially required when estimating all macroscopic material parameters needed in the corresponding macroscopic constitutive model
\end{abstract}

\section{Introduction}

The initiative study on Placa masonry is presented in [1]. Therein, attention was accorded purely to strain based homogenization with emphases on the performance of various damage models. Although allowing for tracking the descending part of the macroscopic stress strain curve when loading the structure beyond its strength limits, it does not provide the expected homogenized macroscopic strength parameters because of the associated constraints generating, e.g., nonzero transverse stresses in case of uniaxial strain. However, the identification of macroscopic counterparts of the parameters of a local constitutive model is crucial owing to an expected computational burden if accounting for every geometrical detail in a macroscopic analysis. This is clear from Fig. 1(c) showing several types of masonry arrangement ranging from a rubble masonry in exterior walls and foundation and a regular masonry used for interior walls. The need for a full-scale analysis of such structures is inevitable due to their high vulnerability to damage when subject to seismic load attributed to the fact that the original design did not reflect any seismic design regulations. The previous study is therefore supported in the present contribution with stress-based homogenization

*Corresponding author: $\underline{\text { sejnom@fsv.cvut.cz }}$ 
strategy $[2,3]$ to properly recover the zero transverse macroscopic stresses in case of uniaxial stress conditions, which in turn are typically assumed when searching for model parameters experimentally.

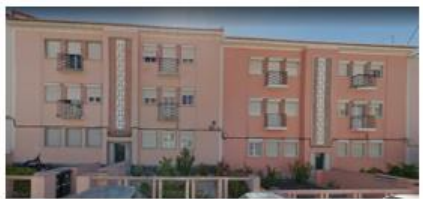

(a)

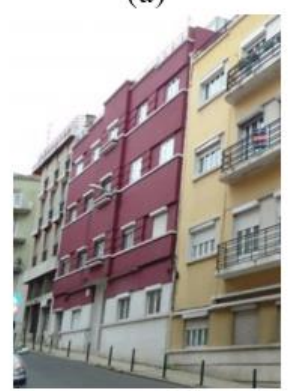

(b)

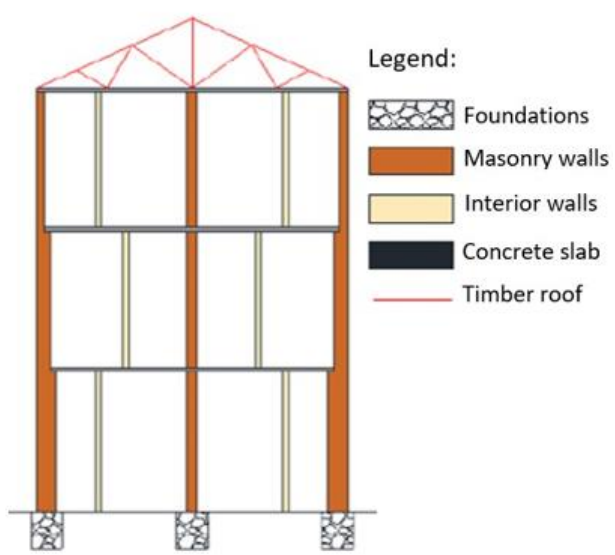

(c)

Fig. 1. (a)-(b) Examples of "Placa" buildings, (c) Scheme of "Placa" building typology with various types of masonry walls.

The paper is organized as follows. Following this introductory part, we shortly address in Section 2 the theoretical grounds of the two constitutive models used in the present study. The set of governing equations arising from the application of finite element method (FEM) within the stress based first order homogenization are presented in Section 3. The results of numerical simulations addressing the influence of both the assumed homogenization strategy and the selected damage model are discussed in Section 4. The principal findings and suggestions for future research directions are finally presented in Section 5.

\section{Damage models}

Limiting attention to the simplest case of a scalar isotropic damage model the local stressstrain relation attains the form

$$
\boldsymbol{\sigma}=(1-\omega) \mathbf{D}_{e} \boldsymbol{\varepsilon}
$$

where $\boldsymbol{\sigma}$ and $\boldsymbol{\varepsilon}$ are the stress and strain vectors, respectively, $\mathbf{D}_{e}$ is the elastic stiffness matrix, and wis the damage parameter, which evolves from 0 for an intact material to 1 for a material volume unable to transfer any stress.

In the present formulation the transition states from 0 to 1 are described by the one dimensional traction-separation law, see Fig. 2, proposed in [4] in the form

$$
\sigma=f_{t} \exp \left(-\frac{\omega_{c r}}{\omega_{c r 0}}\right)
$$

where $f_{t}$ is the tensile strength, $\omega_{c r}$ is the crack opening and $\omega_{c r 0}$ is the parameter controlling the slope of the softening branch. To partially avoid mesh dependency of the results typical of materials with softening the crack opening is smeared over the element as [4]

$$
k-k_{e}=\omega k=\frac{\omega_{c r}}{l_{c h}}
$$




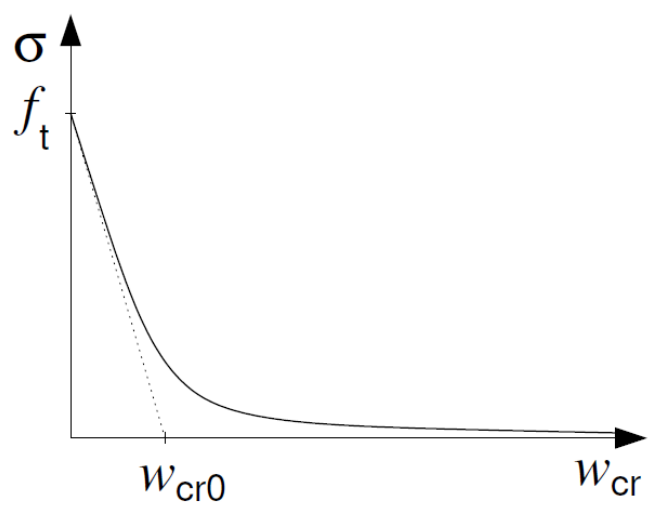

Fig. 2. Softening law.

where the Mazars equivalent strain $k$ is provided by

$$
k=\sqrt{\sum_{\alpha=1}^{3} H\left(\varepsilon_{\alpha}\right)^{2}}
$$

where $H($.$) is the Heaviside function, k_{e}$ is the elastic part of $k$ and $l_{c h}$ is the element characteristic length calculated here as the square root of the element area. Combining a $1 \mathrm{D}$ format of Eq. (1) with Eqs. (3) and (2) and replacing the strain $\varepsilon$ with the maximum equivalent strain in the loading history $\bar{k}$ yields the resulting nonlinear equation to be solved for the damage parameter $\omega$.

$$
(1-\omega) E \bar{k}=f_{t} \exp \left(-\frac{\omega_{l c h} \bar{k}}{u_{f}}\right)
$$

for states where $\bar{k}$ exceeds the elastic threshold $\varepsilon_{0}$.

It is clear that such a model is not very practical for multi-dimensional stress states and generally anisotropic materials as it reduces all components of $\mathbf{D}_{e}$ to zero even in cases when damage evolves in one direction only. It has been clearly shown in [1] that a suitable method of attack is the application of anisotropic [5] or orthotropic damage models. An equivalent format of Eq. (5) written for individual principal strain directions $\alpha$ is provided by

$$
\left(1-D_{\alpha}^{\beta}\right) E\left|\varepsilon_{\alpha}^{\beta}\right|=f_{\beta} \exp \left(-\frac{D_{\alpha}^{\beta} l_{c h}\left|\varepsilon_{\alpha}^{\beta}\right|}{\omega_{c r 0}^{\beta}}\right)
$$

where $D_{\alpha}^{\beta}$ is the damage parameter for $\alpha$ direction with $\beta \in[t, c]$ identifying either tensile or compressive failure. Further details regarding both models can be found in [6] and [7].

\section{Numerical homogenization}

Unlike in [1] we start from the general format of the Hill lemma written as

$$
\left\langle\delta \boldsymbol{\varepsilon}^{T}(\boldsymbol{x}) \Delta \boldsymbol{\sigma}(\boldsymbol{x})\right\rangle=\delta \boldsymbol{E}^{T} \Delta \boldsymbol{\Sigma}
$$

which allows for loading the selected representative volume element (RVE), here assumed in the form of the periodic unit cell (PUC) in Fig. 3(a), by the prescribed macroscopic stress $\boldsymbol{\Sigma}$. Equation (7) states that the volume average of the virtual work done by local strains and 
stresses $(\boldsymbol{\varepsilon}, \boldsymbol{\sigma})$ equals the the virtual work associated with the macroscopic fields, where $\boldsymbol{E}=\langle\boldsymbol{\varepsilon}\rangle$ is the macroscopic strain vector constant over the RVE.

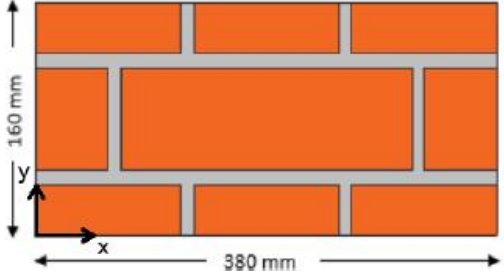

(a)

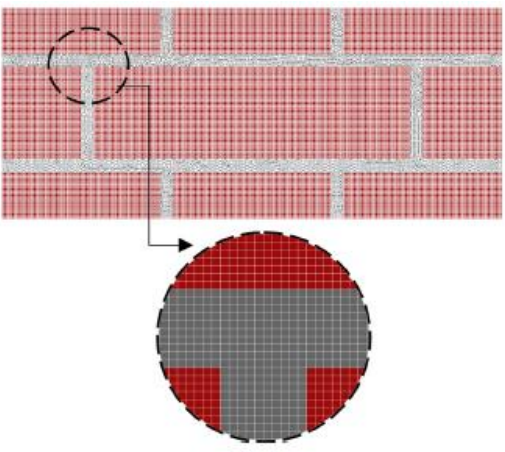

(b)

Fig. 3. (a) PUC representing the "Placa" masonry, (b) Finite element mesh.

Assuming a standard decomposition of the local displacement increment $\Delta u_{i}=\Delta E_{i j} x_{j}+$ $\Delta u_{i}^{*}$ in to macroscopic $\left(\Delta E_{i j} x_{j}\right)$ and fluctuation $\left(\Delta u_{i}^{*}\right)$ parts yields the local strain increment in the form

$$
\Delta \varepsilon(x)=\Delta E+\Delta \varepsilon^{*}(x), \Delta \varepsilon^{*}(x)=\mathbf{B}(x) \Delta r
$$

where Eq. $(8)_{2}$ is the finite element approximation of the fluctuation strain in terms of the nodal displacements, i.e. $\Delta \boldsymbol{u}^{*}=\mathbf{N}(\boldsymbol{x}) \Delta \boldsymbol{r}$, with $\mathbf{N}$ and $\mathbf{B}$ being the standard interpolation and strain matrices, respectively. Substituting Eq. (8) into Eq. (7) gives the final system of algebraic equations for the unknown nodal fluctuation displacements $r$ and macroscopic strain $\boldsymbol{E}$ as

$$
\left[\begin{array}{cc}
\int_{\Omega} \mathbf{L}(\boldsymbol{x}) d \Omega & \int_{\Omega} \mathbf{L}(\boldsymbol{x}) \mathbf{B}(\boldsymbol{x}) d \Omega \\
\int_{\Omega} \mathbf{B}^{T}(\boldsymbol{x}) \mathbf{L}(\boldsymbol{x}) d \Omega & \int_{\Omega} \mathbf{B}^{T}(\boldsymbol{x}) \mathbf{L}(\boldsymbol{x}) \mathbf{B}(\boldsymbol{x}) d \Omega
\end{array}\right]\left\{\begin{array}{c}
\Delta \boldsymbol{E} \\
\Delta \boldsymbol{r}
\end{array}\right\}=\left\{\begin{array}{c}
\Omega \Delta \boldsymbol{\Sigma} \\
\mathbf{0}
\end{array}\right\}
$$

Point out that $\boldsymbol{u}^{*}$ is periodic which in case of rectangular PUC results in the same nodal displacements $\boldsymbol{r}$ on the opposite edges of PUC in Fig. 3(b). Furthermore, if prescribing the macroscopic strain $\Delta \boldsymbol{E}$ we arrive at the strain based formulation adopted in [1]. It is perhaps clear that this more general formulation (9) makes it possible to introduce the mixed loading conditions to drive the analysis in the displacement control regime while keeping the onedimensional format of the macroscopic stress-strain law, e.g. by setting $E_{x x}=\bar{E}, E_{y y}=$ $\Sigma_{x y}=0$ for the state of plane stress. This, however, goes beyond the present scope and will be considered elsewhere.

\section{Numerical simulations}

Similarly to [1], we shall thought the numerical simulations as a substitution for complex laboratory testing. To compare the present results based on Eq. (9) with those derived previously on the basis of strain based formulation we adopt the same computational model as well as the same material properties of the mortar and bricks as used in [1]. Thus the periodic unit cell with the brick layout in Fig. 3(a) typical of "Placa" masonry having the wall thickness of $250 \mathrm{~mm}$, brick nominal dimensions of $250 \times 120 \times 70 \mathrm{~mm}$ and the $10 \mathrm{~mm}$ mortar 
layer will be exploited. The finite element mesh appears in Fig. 3(b). The material parameters are stored in Table 1.

Table 1. Material parameters of brick and mortar.

\begin{tabular}{|c|c|c|c|c|c|c|}
\hline Material & $E[\mathrm{GPa}]$ & $v[-]$ & $f_{t}[\mathrm{MPa}]$ & $G_{f t}[\mathrm{~N} / \mathrm{m}]$ & $f_{c}[\mathrm{MPa}]$ & $\rho\left[\mathrm{kN} / \mathrm{m}^{3}\right]$ \\
\hline Brick & 13.0 & 0.2 & 2.0 & 58.0 & 40.0 & 18.0 \\
\hline Mortar & 0.7 & 0.2 & 0.1 & 10.0 & 16.0 & 17.5 \\
\hline
\end{tabular}

\subsection{Stress based formulation}

In analogy with the simulations presented in [1], we limit again the loading conditions to pure macroscopic tension or compression along the principal directions of material orthotropy $x$ and $y$, recall Fig. 3(a). With reference to the assumed state of plane stress the two loading vectors are

$$
\Sigma_{1}^{T}=\left\{ \pm \Sigma_{x x}, 0,0\right\}, \quad \Sigma_{2}^{T}=\left\{0, \pm \Sigma_{y y}, 0\right\}
$$

The results plotted in Figs. 4 - 7 clearly identify differences between the two damage models suggesting, apart from tension along the y-axis, much higher strength when adopting the orthotropic damage model. This is because in case of tension, Figs. 4 and 5, the orthotropic damage model renders the response in the direction normal to the loading direction essentially elastic, whereas the scalar damage model predicts zero stiffness when $\omega \rightarrow 0$ at a given material point regardless of the loading direction. Note that damage directions (directions of the evolution of damage parameters $\omega$, $\omega$, see e.g. Fig. 4(a,b)) will not necessarily coincide with the principal directions of material orthotropy particularly at the vicinity of brick corners, where the local shear stresses generate rotation of the principal strain directions.

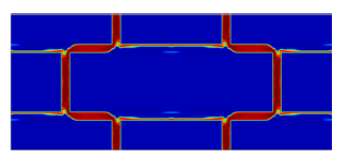

(a)

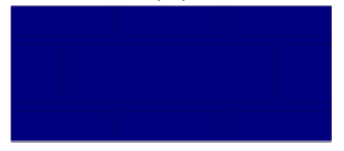

(b)

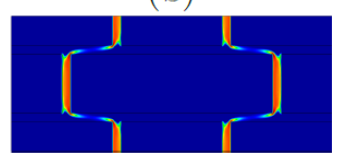

(c)

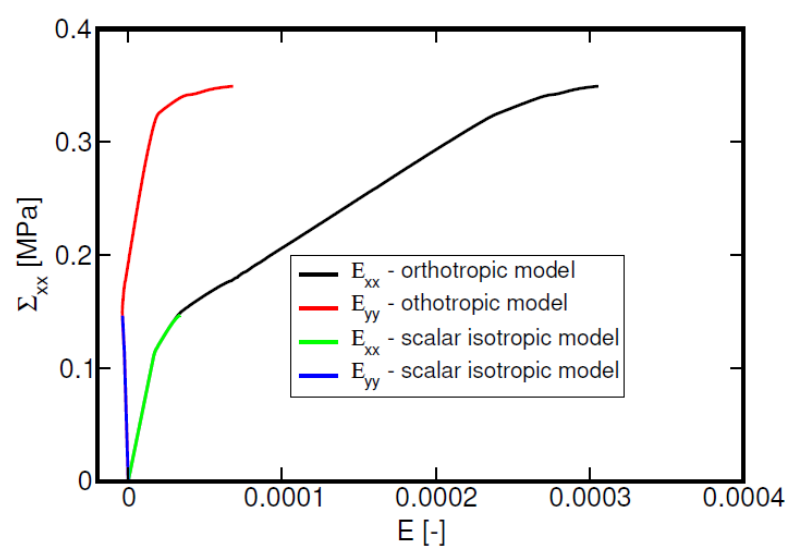

(d)

Fig. 4. Tension along $x$-axis $\left(\Sigma_{x x}>0\right.$ prescribed): (a-c) Damage pattern - (a) orthotropic model $\omega$, (b) orthotropic model $\omega,(\mathrm{c})$ isotropic model $\omega$ (d) Macroscopic stress-strain curve. 


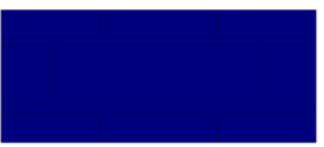

(a)

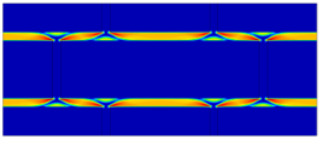

(b)

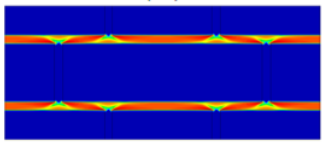

(c)

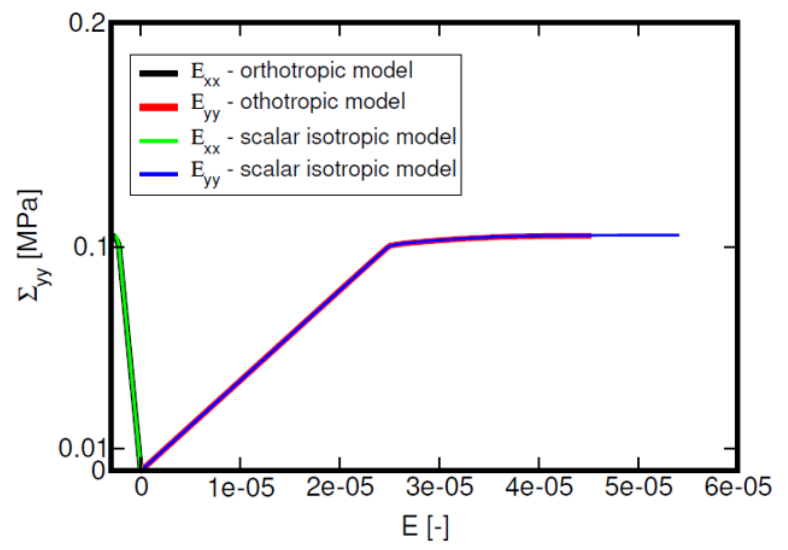

(d)

Fig. 5. Tension along $y$-axis $\left(\Sigma_{y y}>0\right.$ prescribed): (a-c) Damage pattern - (a) orthotropic model $\left.\omega\right),(b)$ orthotropic model $\omega,(\mathrm{c})$ isotropic model $\omega(\mathrm{d})$ Macroscopic stress-strain curve.

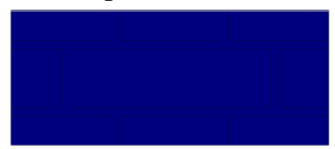

(a)

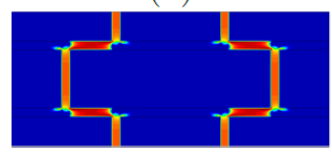

(b)

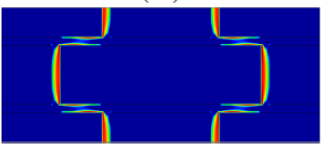

(c)

\section{E [-]}

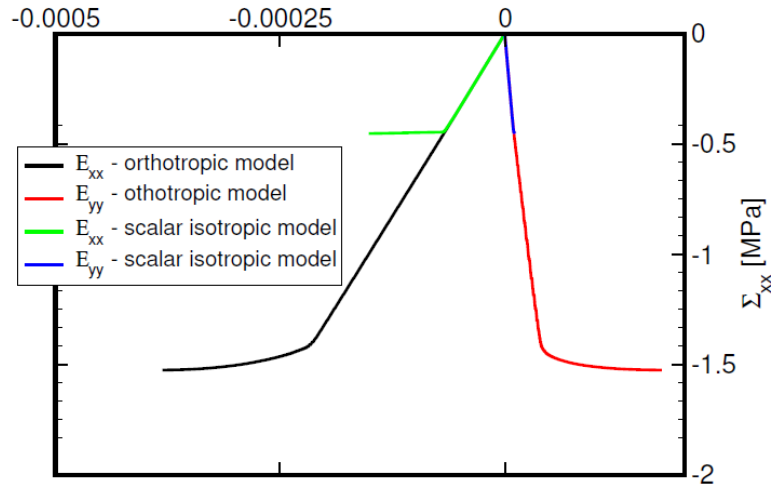

(d)

Fig. 6. Compression along x-axis $\left(\Sigma_{y y}<0\right.$ prescribed): (a-c) Damage pattern - (a) orthotropic model $\omega$, (b) orthotropic model $\omega$, (c) isotropic model $\omega$ (d) Macroscopic stress-strain curve.

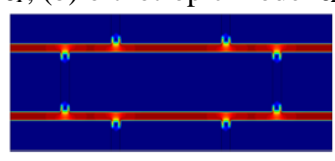

(a)

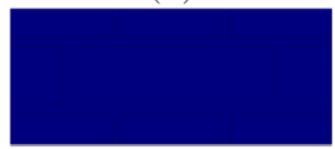

(b)

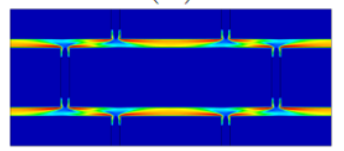

(c)

\section{$E[-]$}

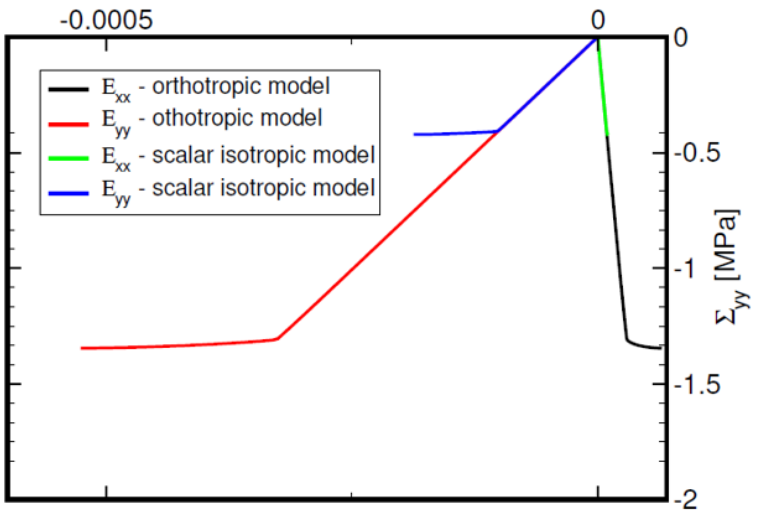

(d)

Fig. 7. Tension along $x$-axis $\left(\Sigma_{y y}<0\right.$ prescribed): (a-c) Damage pattern - (a) orthotropic model $\omega$, (b) orthotropic model $\omega,(\mathrm{c})$ isotropic model $\omega$ (d) Macroscopic stress-strain curve. 
When comparing the response in tension and compression along the same direction, Figs. 4 and 6 and Figs. 5 and 7, it is worth pointing out that the compressive loading generates local tensile stresses in the transverse direction causing failure in tension, which explains seemingly similar damage patterns for tension and compression. Nevertheless, the onset of damage appears, as expected, at different levels of the applied stress.

The need for properly reflecting the actual brick layout is evident from Fig. 5 showing an identical response predicted by both models. This is not surprising once realizing that in tension applied along the $y$-axis the weak mortar behaves as the weakest link. So, in this case, the stiffness along the $x$-axis, although keeping essentially the original value in the case of orthotropic model, provides no support. Similar results have already been observed in [1] focusing on the strain based formulation with the macroscopic strain prescribed.

\subsection{Comparing stress and strain based formulations}

The present section serves to compare the macroscopic response provided by both homogenization formulations. The results appear in Figs. 8 and 9 for tension and compression, respectively.

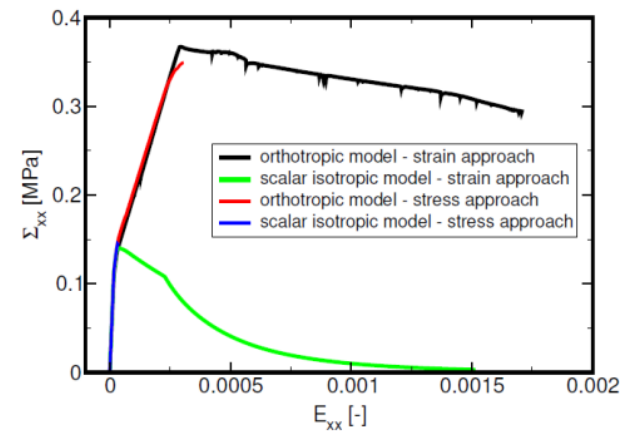

(a)

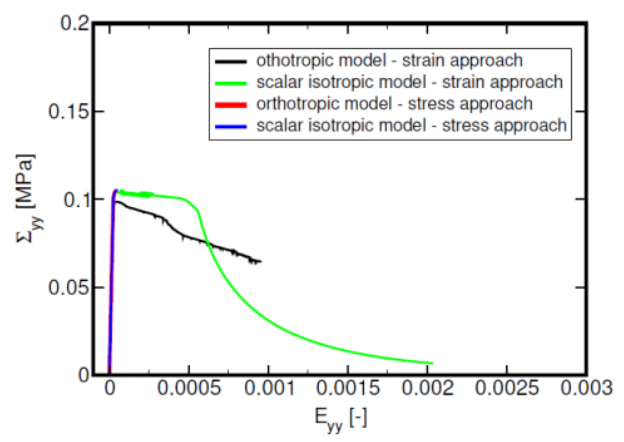

(b)

Fig. 8. Comparing tensile response based on strain $\left(E_{i i}>0\right.$ prescribed $)$ and stress $\left(\Sigma_{i i}>0\right.$ prescribed $)$ based formulation: (a) $x$-direction, (b) $y$-direction.

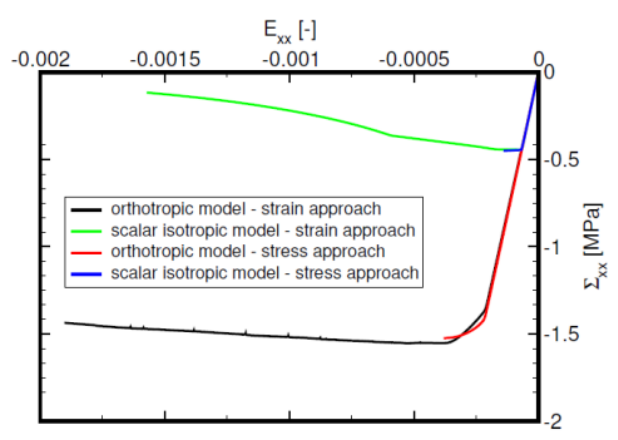

(a)

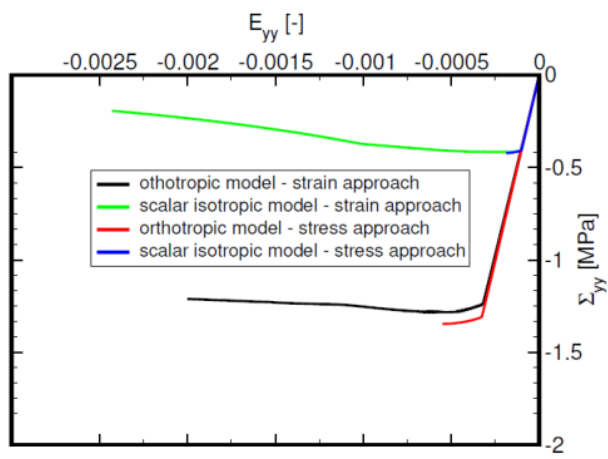

(b)

Fig. 9. Comparing tensile response based on strain $\left(E_{i i}>0\right.$ prescribed $)$ and stress $\left(\Sigma_{i i}>0\right.$ prescribed $)$ based formulation: (a) $x$-direction, (b) $y$-direction. 
It is seen that the two approaches predict only a slightly different value of the peak load albeit to different constraints attributed to particular loading conditions. While the stress based formulation generates the true uniaxial stress state as one would expect in laboratory for an unconstrained material, the strain based approach results in zero transverse strains and nonzero transverse stresses for uniaxial extension/compression. The second observation promotes the strain based approach, as essentially compatible with displacement control type of loading, once requesting the response beyond the peak load. This is obviously not possible with the stress based approach.

\section{Conclusions}

The present paper was concerned with the prediction of macroscopic response of the type of masonry structure observed for "Placa" buildings. Following our previous study presented in [1] we turned our attention to stress based formulation, which allows for reflecting the same type of macroscopic state of stress as one would observe in laboratory.

While the derived results supported most of the findings discussed in [1] with regard to the two adopted damage models, they also confirmed the expected inability of the stress based approach to track the descending part of the macroscopic stress-strain curve. This, however, is feasible with the strain based approach as evident from Figs. 8 and 9. Thus to exploit advantages of individual approaches the formulation based on the mixed boundary/loading conditions will be examined in our future research.

The financial support by the Czech Grant Agency GAČR, project No. 18-24867S, is gratefully acknowledged.

\section{References}

1. V. Bernardo, T. Krejčí, T. Koudelka, M. Šejnoha, Acta Polytechnica (2019)

2. J. C. Michel, H. Moulinec, P. Suquet, Computer Methods in Applied Mechanics and Engineering 172 (1999) 109-143. (2009)

3. M. Šejnoha, J. Zeman, Micromechanics in Practice, WIT Press, Southampton, Boston, (2013)

4. Z. P. Bažant, B. H. Oh, Crack band theory for fracture of concrete, Matériaux et Construction 16 (3) (1983) 155-177

5. E. Papa, A. Taliercio, Anisotropic Damage Model for the Multiaxial Static and Fatigue Behaviour of Plain Concrete, Engineering Fracture Mechanics

6. T. Krejčí, T. Koudelka, J. Šejnoha, J. Zeman, Civil-Comp Press (2009), paper 131

7. T. Koudelka, T. Krejčí, J.Šejnoha, Civil-Comp Press, (2009), paper 132. 